\title{
Modeling and Quantification of Electrochemical Reactions in RDE (Rotating Disk Electrode) and IRDE (Inverted Rotating Disk Electrode) Based Reactors
}

\author{
Lucía Fernández Macía, Heidi Van Parys, Tom Breugelmans, \\ Els Tourwé and Annick Hubin \\ Electrochemical and Surface Engineering Group, Vrije Universiteit Brussel \\ Belgium
}

\section{Introduction}

Whether it is for the design of a new electrochemical reactor or the optimization of an existing electrochemical process, it is of primordial importance to have the possibility to predict the behavior of a system. For example, the process of electrogalvanization of steel on an industrial scale would not be possible without knowing the main and side reactions taking place during the deposition of zinc on the metallic surface. However, understanding their mechanism is only the first point. The characteristic parameters of the reaction need to be identified and quantified in order to obtain a correct reactor design and to achieve the optimal operating conditions. Nowadays, part of the technological know-how still relies on best practice guidance, most often gained from years of experience with trial and error. Condensing those findings into empirical models may help to control some of the process parameters and make predictions of the system behavior within a small operation window. The problem, however, is that such a model acts as a black box and that a profound comprehension of the physical and electrochemical phenomena will fail to come.

The aim of a kinetic study is the determination of the mechanism of the electrochemical reaction and the quantification of its characteristic parameters: charge transfer parameters (rate constants and transfer coefficients) and mass transfer parameters (diffusion coefficients). Nevertheless, determining kinetic parameters accurately from the experimental results remains complex.

Linear sweep voltammetry (LSV) in combination with a rotating disk electrode (RDE) is a widely used technique to study electrode kinetics. Different methods exist to extract the values of the process parameters from polarization curves. The Koutecky-Levich graphical method is frequently used to determine the mass transfer parameters (Diard et al., 1996) : the slope of a plot of the inverse of the limiting current versus the inverse of the square root of the rotation speed of the rotating disk electrode is proportional to the diffusion coefficient. If more than one diffusing species is present, this method provides the mean diffusion coefficient of all species. The charge transfer current density is determined from the inverse of the intercept. In practical situations, however, the experimental observation of a limiting current 
can sometimes be masked by other reactions, e.g., in (Gattrell et al., 2004), and in that case Koutecky-Levich method cannot be used.

Also, to calculate the charge transfer parameters, a plot of the natural logarithm of the charge transfer current density as a function of potential, known as a Tafel plot, is often constructed. In the linear region of this curve, the transfer coefficient can be deduced from the slope and the rate constants from the intercept. The Tafel method is well established for simple reaction mechanisms (Bamford \& Compton, 1986; Diard et al., 1996), but it becomes much more complicated for complex mechanisms (Gattrell et al., 2004; Wang et al., 2004). When there are significant diffusional or ohmic effects in the electrolyte, or additional electrode reactions, the Tafel plot deviates from linearity (Yeum \& Devereux, 1989) and the charge transfer parameters cannot be determined.

Besides these well-known graphical methods, some authors suggest other methods to extract the kinetic parameters from an LSV experiment. They usually involve the fitting of theoretical expressions to the experimental data. In (Rocchini, 1992) the charge transfer parameters are estimated by fitting experimental polarization curves with exponential polynomials. Obviously, this method is only valid if the reaction rate is determined by charge transfer alone. Caster et al. fit convolution potential sweep voltammetry experiments with equations for a reversible charge transfer reaction with only one reactant present initially and under conditions of planar diffusion (Caster et al., 1983). No other steps are allowed to occur either before or after the electrode reaction. Yeum and Devereux propose an iterative method for fitting complex electrode polarization curves (Yeum \& Devereux, 1989). They split up the total current density into contributions from the partial reactions and use simplified expressions for the current-potential relations. With these expressions they try to find the parameters that optimize the correlation between model and experimental data by minimizing a least squares cost function. This optimization is done by trial-and-error. In (Rusling, 1984) tabulated dimensionless current functions are fitted to linear sweep voltammograms. Therefore, a least squares cost function is minimized; however, no details on the minimizing algorithm are given.

In a series of papers, Harrison describes a hardware/software system for the complete automation of electrode kinetic measurements (Aslam et al., 1980; Cowan \& Harrison, 1980a;b; Denton et al., 1980; Harrison, 1982a;b; Harrison \& Small, 1980a;b). This involves the fitting of the data using a library of reaction schemes to determine the model parameter values. A quasi-Newton method is used to minimize the modulus of the differences between experiment and theory or the sum of the weighted squares of the differences. Although it is emphasized that care has to be taken in weighting the observations, no information on the determination of the weighting factors is given. Moreover, no criterion to decide whether the fitting is acceptable or not is discussed.

In (Bortels et al., 1997; Van den Bossche et al., 1995; 2002; Van Parys et al., 2010) a numerical approach is developed in order to define the underlying reaction mechanism. By using the MITReM (Multiple Ion Transport and Reaction Model) model, mass transport by convection, diffusion and migration but also the presence of homogeneous reactions in the electrolyte, are accounted for. The related model parameters such as diffusion coefficients, rate constants and transfer coefficients are adjusted in order to improve the agreement between experimental and simulated polarization curves. Thus, the best parameter values, corresponding to the best simulated curve, are selected by a chi-by-eye approach, without a statistical evaluation. 
Although the reaction and transport models are defined more precisely, the lack of a fitting tool does not allow a reliable determination of the model parameters.

A quantitative, accurate and statistically founded modeling approach of electrochemical reactions has been the focus of an extensive work in our research group (Aerts et al., 2011; Tourwé et al., 2007; 2006; Van Parys et al., 2008). It is a generally applicable method to model an electrochemical reaction and to determine its mass and charge transfer parameters quantitatively. The reliability of the model parameters and the accuracy of the parameter fitting are key-elements of the method. A plausible reaction mechanism and the characteristic parameters of the electrochemical reaction are extracted from LSV experiments with a rotating disk electrode. Compared to others, this method offers the advantage that it uses one integrated expression that accounts for mass and charge transfer steps, and this without simplifying their mathematical expressions. The whole polarization curve is considered, rather than just some part in which only mass or charge transfer are supposed to be rate determining.

In this paper, we explain throughly this modeling methodology for the rotating disk electrode (RDE) and the inverted rotating disk electrode (IRDE) configurations. The modeling and quantification of the electrochemical parameters are applied to redox reactions with one electron transfer mechanism: the ferri/ferrocyanide system and the hexaammineruthenium (III)/(II) system.

\section{Linear sweep voltammetry in combination with a rotating disk electrode}

Linear sweep voltammetry with a rotating disk electrode (LSV/RDE) is a powerful technique for providing information on the mechanism and kinetics of an electrochemical reaction. Since the current density is a measure for the rate of an electrochemical reaction, LSV provides a stationary method to measure the rate as a function of the potential. In other words, the technique is used to distinguish between the elementary reactions taking place at the electrode as a function of the applied potential. Different elementary steps are often coupled, however, the overall current is determined by the slowest process (rate determining step). As a steady state technique, linear sweep voltammetry can only give mechanistic information about rate determining elementary reactions.

To determine a quantitative model for an electrochemical process, first a plausible reaction model is proposed and afterwards combined with a transport model. The combination of both models enables the formulation of the mass balances of the species and the conservation laws, which results in a set of non-linear partial differential equations, where the electrochemical reactions constitute a boundary condition at the electrode. While the reaction model is proper to the reaction under study, the transport model is merely determined by the mass transport of the species in the electrochemical reactor. As a result, it is possible to direct an electrochemical investigation in an adapted experimental reactor (electrochemical cell) under conditions for which the description of the transport phenomena can be simplified, without a loss of precision.

For controlling the mass transport contribution to the overall electrochemical kinetics, a rotating disk electrode possesses favorable features. The RDE configuration provides analytical equations to describe the mass transport and hydrodynamics in the electrochemical cell. It is known that a simplified transport model can be used if an RDE and diluted solutions are used in the experimental set-up. The hydrodynamic equations and the 
convective-diffusion equation for a rotating disk electrode have been solved rigorously for the steady state (Levich, 1962; Slichting, 1979). The axial symmetry of the configuration of the RDE reactor and the uniform current distribution allow a one-dimensional description. Moreover, at sufficient flow rate (when natural convection can be ignored), the hydrodynamics in diluted solution are not influenced by changes in concentrations due to electrochemical reactions. The mathematical problem can thus be solved more easily. Levich reduced the equation of convection transport to an ordinary differential equation (Albery \& Hitchman, 1971; Levich, 1962; Slichting, 1979).

To model an electrochemical reaction and determine its mass and charge transfer parameters quantitatively, an electrochemical data fitting tool has been developed in our research group. From an analytical approach, it is designed to extract a quantitative reaction mechanism from polarization curves.

\section{Analytical fitting of electrochemical parameters}

The proposed analytical modeling of electrochemical reactions is founded on four building blocks. Figure 1 illustrates the structure of the modeling methodology.

The results of the experimental study These are the current-potential couples defining the polarization curve. The mean of multiple experiments that are performed under identical conditions is the experimental data for the modeling. The standard deviation of the experiments is used in the fitting procedure.

The mathematical expression for the proposed model The proposed model is based on well-considered reaction and transport models for the studied reaction. The mathematical expression of the reaction-transport model is derived from the basic equations that describe what happens during an electrochemical reaction. It has the following form: current = function (potential, experimental parameters, model parameters), where the experimental parameters describe the experimental conditions, like e.g. temperature, rotation speed of the RDE, concentration, ..., and the model parameters are the unknown parameters that need to be quantitatively determined, like e.g. rate constants, transfer coefficients, ....

The fitting procedure In this block the differences between experimental and theoretical data are minimized. A weighted least squares cost function is formulated. The Gauss-Newton and Levenberg-Marquardt method are implemented to minimize this cost function and eventually provide the parameter values which best describe the data. Moreover, the standard deviations of the estimated parameters are also calculated.

A statistical evaluation If a statistical evaluation of the fitting results demonstrate a good description of the experiment by the model, a quantitative reaction mechanism is obtained. If, on the other hand, no good agreement between experiment and model is achieved, a new mechanism has to be proposed and the previous steps should be repeated.

\subsection{The mathematical expression for the proposed reaction and transport models}

Once the reaction model is defined, the transport model must be included. Using an RDE as the working electrode for the LSV experiments, the transport equations can be simplified. In addition, assuming that the electrolyte is a diluted solution, the migration term in the transport model can be neglected. This section provides the basic equations for mass and 


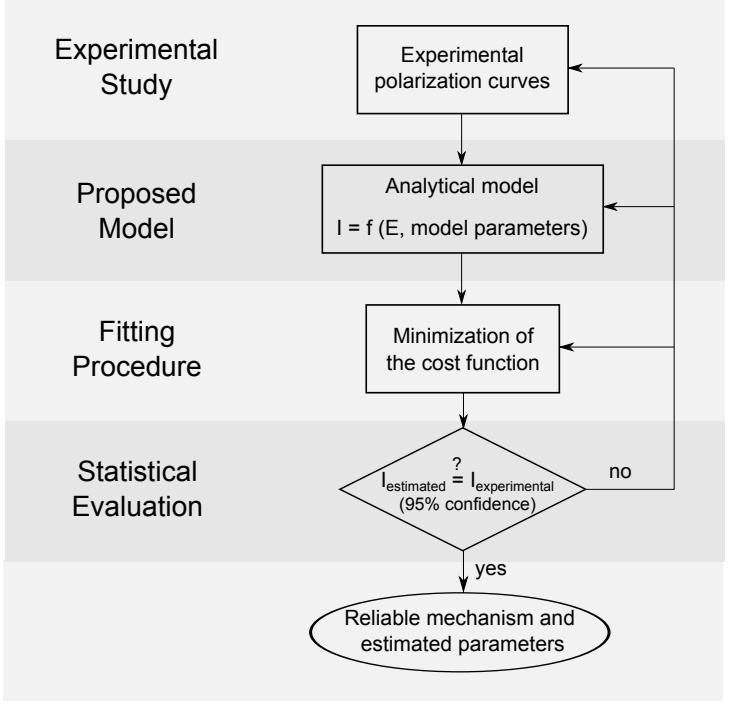

Fig. 1. The four building blocks of the modeling methodology.

charge transfer, which can be found in numerous textbooks (Bamford \& Compton, 1986; Diard et al., 1996; Newman, 1973; Pletcher, 1991; Thirsk \& Harrison, 1972; Vetter, 1967).

Consider a uniformly accessible planar electrode, immersed in an electrolyte that contains electroactive species and an excess of inert supporting electrolyte. At the surface an electrochemical reaction is taking place, which has $P$ partial heterogeneous electrochemical or chemical reactions with $N_{v}$ electroactive species in the electrolyte or in the electrode material and $N_{s}$ electroactive species present in an adsorbed phase on the electrode surface. $N$ is the total number of electroactive species involved in the reaction: $N=N_{v}+N_{s}$. The $j^{\text {th }}$ step of the reaction can be written as:

$$
\begin{gathered}
K_{j} \\
\sum_{i=1}^{N} r_{i j} X_{i} \leftrightarrow \sum_{i=1}^{N} p_{i j} X_{i} \pm n_{j} e \\
K_{j}^{\prime}
\end{gathered}
$$

with $r_{i j}$ and $p_{i j}$ the stoichiometric coefficients, the index $i$ refers to the considered species, the index $j$ to the partial reaction. $K_{j}$ and $K_{j}^{\prime}$ are the rate constants. For an electrochemical reaction, they depend on the electrode potential. $n_{j}$ is the number of electrons exchanged in the $j^{\text {th }}$ partial reaction. For an electrochemical reaction $n_{j}$ is preceded by a plus sign if the reaction is written in the sense of the oxidation and by a minus sign if written in the sense of the reduction. For a chemical reaction $n_{j}$ equals zero.

The global reaction is described by the relations that connect the electrode potential $E$ to the faradaic current density $i_{f}$, the interfacial concentrations of the volume species $X_{i}(0)$ and the 
surface concentrations $X_{i}$ of the adsorbed species ${ }^{1}$. Under steady-state conditions and using an $\mathrm{RDE}$, the general system of equations describing this electrochemical systems is given by:

- the rate $\left(v_{j}\right)$ expressions for each partial reaction:

$$
v_{j}=K_{j} \prod_{i=1}^{N} X_{i}^{r_{i j}}-K_{j}^{\prime} \prod_{i=1}^{N} X_{i}^{p_{i j}}
$$

$X_{i}$ is the concentration of the electroactive species. In electrode kinetics, concentrations are usually employed rather than activities and thus the rate constants include the product of activity coefficients. $K_{j}$ and $K_{j}^{\prime}$ are the potential dependent rate constants of the partial reaction, given by:

$$
\begin{aligned}
& K_{j} \text { or } K_{j}^{\prime}=k_{o x, j} \exp \left(\frac{\alpha_{o x, j} n_{j} F E(t)}{R T}\right) \\
& K_{j} \text { or } K_{j}^{\prime}=k_{r e d, j} \exp \left(\frac{-\alpha_{r e d, j} n_{j} F E(t)}{R T}\right)
\end{aligned}
$$

with $\alpha_{o x, j}+\alpha_{r e d, j}=1$. F is the Faraday constant $(96485 \mathrm{C} / \mathrm{mol}), \mathrm{R}$ is the ideal gas constant $(8.32 \mathrm{~J} / \mathrm{mol} \mathrm{K})$ and $\mathrm{T}$ is the absolute temperature $(K) . k_{o x, j}$ is the rate constant of the partial reaction $j$ in the sense of the oxidation and $k_{r e d, j}$ is the one in the sense of the reduction. $\alpha_{o x, j}$ and $\alpha_{r e d, j}$ are the transfer coefficients in the sense of the oxidation and reduction respectively. They are supposed to be independent of the electrode potential (Diard et al., 1996). If adsorbed species are involved in the electrochemical reaction the rate constants $K_{j}$ and $K_{j}^{\prime}$ may depend on the coverage.

- the relations that connect the faradaic current density to the rates of the partial electrochemical reactions:

$$
i_{f}=F \sum_{j=1}^{P} s_{e j} v_{j}
$$

where $s_{e j}= \pm n_{j}$. The + or - sign is fixed by the following convention: an oxidation current is counted positive, a reduction current negative.

- the relations expressing the transformation rate of the electroactive species at the interface electrode/electrolyte, and the continuity relation which expresses that this transformation rate is equal to the interfacial mass transport flux of the species $X_{i}$ :

$$
\begin{aligned}
v_{X_{i}} & =J_{X_{i}} \\
& =-m_{X_{i}}\left[X_{i}^{*}-X_{i}(0)\right] \\
& =\sum_{j=1}^{P} s_{i j} v_{j} \\
i & =1, \ldots, N_{v}
\end{aligned}
$$

\footnotetext{
${ }^{1}$ To simplify the notation the symbol $X_{i}$ is used to describe the chemical species in a reaction and its concentration $X_{i}=\left[X_{i}\right]$
} 
or

$$
\begin{aligned}
v_{X_{i}}(t) & =0 \\
i & =N_{v}+1, \ldots, N
\end{aligned}
$$

were $J_{X_{i}}$ is the molecular flux (expressed in $\mathrm{mol} / \mathrm{m}^{2} \mathrm{~s}$ ) of $X_{i}$, equal to the number of moles of $X_{i}$ going per unit of time across a unit plane, perpendicularly oriented to the flow of the species. $X_{i}^{*}$ is the bulk concentration of species $X_{i} \cdot m_{X_{i}}$ is the mass transport rate constant for diffusion and convection of species $X_{i}$, given by:

$$
m_{X_{i}}=0.620 D_{X_{i}}^{2 / 3} v^{-1 / 6} \omega^{1 / 2}
$$

with $D_{X_{i}}$ the diffusion coefficient of $X_{i}, v$ the kinematic viscosity and $\omega$ the rotation speed of the RDE.

At the maximum diffusion rate, the concentration at the electrode is zero and the limiting current is achieved. The Levich equation provides an expression of the limiting current as a function of the mass transport rate constant and the bulk concentration:

$$
I_{\text {lim }}=0.620 n F S D_{X_{i}}^{2 / 3} v^{-1 / 6} \omega^{1 / 2} X_{i}^{*}
$$

with $S$ the electrode surface and $n$ the number of electrons exchanged in the reaction. This equation thus applies to the totally mass-transfer-limited condition at the RDE.

\subsection{The fitting procedure}

Many comprehensive textbooks about parameter estimation and minimization algorithms are available. The development of the fitting procedure for the analytical modeling of electrochemical reactions is founded on a few of them (Fletcher, 1980; Kelley, 1999; Norton, 1986; Pintelon \& Schoukens, 2001; Press et al., 1988; Sorenson, 1980).

Given a set of observations, one often wants to condense and summarize the data by fitting it to a 'model' that depends on adjustable parameters. In this work the 'model' is the current-potential relation, describing the polarization curve, which is derived from the basic laws for mass and charge transfer (given in section 3.1). The fitting of this mathematical expression provides the values of characteristic parameters (rate constants, transfer coefficients, diffusion coefficients), resulting in a quantitative reaction mechanism for the electrochemical reaction.

The basic approach is usually the same: a cost function that measures the agreement between the data and the model with a particular choice of parameters is designed. The cost function usually defines a distance between the experimental data and the model and is conventionally arranged so that small values represent close agreement. The parameters of the model are then adjusted to achieve a minimum in the cost function. This is schematically illustrated in Figure 2. It shows an imaginary experiment, modeled with the well-known Butler-Volmer equation, which describes the rate of an electrochemical reaction under charge transport control. By changing the values of the transfer coefficients and the rate constants, the distance between model and experiment varies for each data point. The cost function takes all these distances into account. The estimates or best-fit-parameters are the arguments that minimize the cost function. 


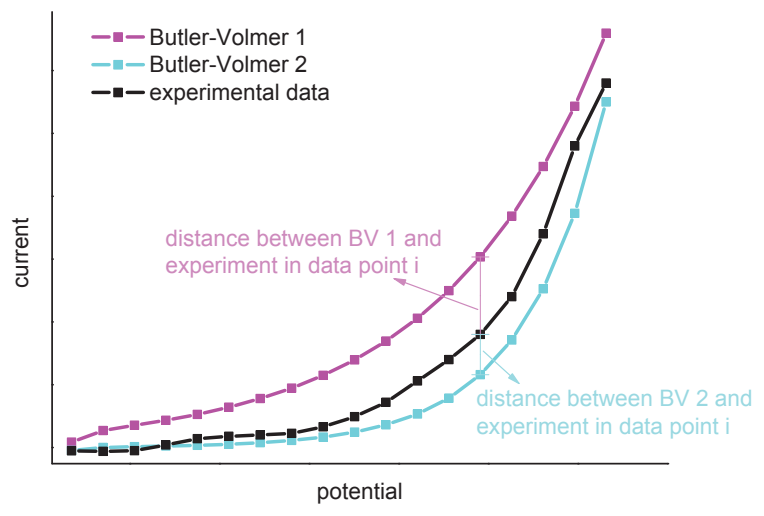

Fig. 2. The cost function as a measure of the agreement between the experiment and the model.

In most data fitting problems the match between the model and the measurements is quantified by a least squares cost function. Consider a multiple input, single output system modeled by $y_{0}(l)=F\left(u_{0}(l), \theta\right)$ with $l$ the measurement index, $y(l) \in \mathbb{R}, u(l) \in \mathbb{R}^{1 \times M}$, and $\theta \in \mathbb{R}^{n_{\theta} \times 1}$ the parameter vector. The aim is to estimate the parameters from noisy observations of the output of the system: $y(l)=y_{0}(l)+n_{y}(l)$. This is done by minimizing the sum of the squared errors, $e(l)=y(l)-y_{0}(l)$ :

$$
\hat{\theta}_{L S}=\underset{\theta}{\arg \min } V_{L S}(\theta) \text { with } V_{L S}=\frac{1}{2} \sum_{l=1}^{N_{d p}} e^{2}(l)
$$

with $N_{d p}$ the number of data points. $\arg \min _{\theta}$ stands for: "find the argument $\theta$ that minimizes ...".

In this work polarization curves will be modeled. The current response (= output $y$ ) to a linearly changing potential (= input $u_{0}$ ) is measured in $N_{d p}$ different points (with index $l$ ) and the transfer coefficients, rate constants and diffusion coefficients $(=\theta)$ need to be estimated. The function $F$ is a theoretical expression which relates the current to the potential and the model parameters.

The Gauss-Newton algorithm is very well suited to deal with the least squares minimization problem. The numerical solution is found by applying the following iterative process:

1. Solve $J\left(\theta^{(k)}\right)^{T} J\left(\theta^{(k)}\right) \delta^{(k)}=-J\left(\theta^{(k)}\right)^{T} e\left(\theta^{(k)}\right)$

2. Set $\theta^{(k+1)}=\theta^{(k)}+\delta^{(k)}$

with $J(\theta)$ the $N_{d p} \times n_{\theta}$ Jacobian matrix. This is the matrix of all first-order partial derivatives of the error $e$. To improve the numerical conditioning of the expression 1 of the iterative process, the Jacobian can be scaled by multiplying each column with the corresponding parameter value from the previous iteration. As the Gauss-Newton method is an iterative method, it 
generates a sequence of points, noted as $\theta^{(1)}, \theta^{(2)}, \theta^{(3)}, \ldots$ or $\left\{\theta^{(k)}\right\}$. The iteration mechanism stops when a convergence test is satisfied, and the following criterion is used here:

$$
\left|\frac{\theta_{i}^{(k+1)}-\theta_{i}^{(k)}}{\theta_{i}^{(k+1)}}\right| \leq \epsilon_{i} \forall i
$$

where $\epsilon_{i}$ is the relative error in the minimization algorithm, which is defined by the user. That means that the fitting stops when the parameter values from one iteration to another do not change more than a low $\epsilon_{i}$ value.

Using singular value decomposition (SVD) techniques, the Gauss-Newton algorithm can be solved without forming the product $J\left(\theta^{(k)}\right)^{T} J\left(\theta^{(k)}\right)$ so that more complex problems can be solved because the numerical errors are significantly reduced. By SVD the matrix $J$ is transformed into the product $J=U \Sigma V^{T}$ with $U$ and $V$ orthogonal matrices: $U^{T} U=I$ and $V^{T} V=V V^{T}=I . \Sigma$ is a diagonal matrix with the singular values on the diagonal. This leads to the following expression for $\delta$ :

$$
\delta=-V \Sigma^{-1} U^{T} e
$$

Under quite general assumptions on the noise, some regularity conditions on the model $F\left(u_{0}(l), \theta\right)$ and the excitation (choice of $u_{0}(l)$ ), consistency ${ }^{2}$ of the least squares estimator is proven. Asymptotically (for the number of data points going to infinity) the covariance matrix $C_{L S}$ of the estimated model parameters is given by:

$$
C_{L S}=\left(J\left(\theta^{(k)}\right)^{T} J\left(\theta^{(k)}\right)\right)^{-1} J\left(\theta^{(k)}\right)^{T} C_{y} J\left(\theta^{(k)}\right)\left(J\left(\theta^{(k)}\right)^{T} J\left(\theta^{(k)}\right)\right)^{-1}
$$

with $C_{y}=\operatorname{cov}\left\{n_{y}\right\}$ the covariance matrix of the noise.

The Levenberg-Marquardt algorithm is a popular alternative to the Gauss-Newton method. This method is often considered to be the best type of method for non-linear least squares problems, but the rate of convergence can be slow. The iterative process is given by:

1. Solve $\left(J\left(\theta^{(k)}\right)^{T} J\left(\theta^{(k)}\right)+\lambda^{2} I\right) \delta^{(k)}=-J\left(\theta^{(k)}\right)^{T} e\left(\theta^{(k)}\right)$

2. Set $\theta^{(k+1)}=\theta^{(k)}+\delta^{(k)}$

or using SVD:

$$
\delta=-V\left(\begin{array}{ccc}
\frac{s_{1}}{s_{1}^{2}+\lambda^{2}} & 0 & 0 \\
0 & \frac{s_{2}}{s_{2}^{2}+\lambda^{2}} & 0 \\
0 & 0 & \ddots
\end{array}\right) U^{T} e
$$

with $s$ the singular values and $\lambda$ is called the Levenberg-Marquardt factor. As starting value $\lambda=\frac{s_{1}}{100}$ is chosen. If the value of the cost function decreases after performing an iteration, a new iteration is performed with $\lambda_{\text {new }}=\lambda_{\text {old }} * 0.4$. If the cost function increases, $\lambda_{\text {new }}=$ $\lambda_{\text {old }} * 10$ is chosen and the old value of $\theta$ is maintained.

Some numerical aspects of these methods are also worth mentioning. Whether the Gauss-Newton or the Levenberg-Marquardt algorithm is used, the expression for equation to be solved is always written as:

\footnotetext{
$\overline{2}$ An estimator $\hat{\theta}$ is strongly consistent if it converges almost surely to $\theta_{0}$ : a.s. $\lim _{N_{d p} \rightarrow \infty} \hat{\theta}\left(N_{d p}\right)=\theta_{0}$, with $\theta_{0}$ the true (unknown) value $\theta$.
} 


$$
X \delta=e
$$

Consequently, a change $\Delta e$ of $e$, also causes $\delta$ to change by $\Delta \delta$. The problem is said to be well conditioned if a small change of $e$ results in a small change of $\delta$. If not, the problem is ill-conditioned. It can be shown that

$$
\frac{\|\Delta \delta\|_{2}}{\|\delta\|_{2}} \leq \frac{s_{\max }}{s_{\min }} \frac{\|\Delta e\|_{2}}{\|e\|_{2}}
$$

with \|\|$_{2}$ the 2-norm given by:

$$
\|x\|_{2}=\sum_{i} x_{i}^{2}
$$

and $\kappa=\frac{s_{\max }}{s_{\min }}$, the ratio of the largest singular value to the smallest, is called the condition number of $X$. If $\kappa$ is large, the problem is ill-conditioned.

In Eq. (9) all measurements are equally weighted. In many problems it is desirable to put more emphasis on one measurement with respect to the other. This is done to make the difference between measurement and model smaller in some regions. If the covariance matrix of the noise is known, then it seems logical to suppress measurements with high uncertainty and to emphasize those with low uncertainty. In practice it is not always clear what weighting should be used. If it is, for example, known that model errors are present, then the user may prefer to put in a dedicated weighting in order to keep the model errors small in some specific operation regions instead of using the weighting dictated by the covariance matrix.

In general, the weighted least squares estimator $\hat{\theta}_{W L S}$ is:

$$
\hat{\theta}_{W L S}=\underset{\theta}{\arg \min } V_{W L S}(\theta) \text { with } V_{W L S}=\frac{1}{2} e(\theta)^{T} W e(\theta)
$$

where $W \in \mathbb{R}^{N \times N}$ is a symmetric positive definite weighting matrix. All the remarks on the numerical aspects of the least squares estimator are also valid for the weighted least squares. This can be easily understood by applying the following transformation: $\epsilon=S e$ with $S^{T} S=$ $W$ so that $V_{W L S}=\epsilon^{T} \epsilon$, which is a least squares estimator in the transformed variables. This also leads to the following Gauss-Newton algorithm to minimize the cost function:

$$
\theta^{(k+1)}=\theta^{(k)}+\delta^{(k)}, \text { with } J\left(\theta^{(k)}\right)^{T} W J\left(\theta^{(k)}\right) \delta^{(k)}=-J\left(\theta^{(k)}\right)^{T} W e\left(\theta^{(k)}\right)
$$

Eq. (12) is generalized to (noticing that $W^{T}=W$ ):

$$
C_{W L S}=\left(J\left(\theta^{(k)}\right)^{T} W J\left(\theta^{(k)}\right)\right)^{-1} J\left(\theta^{(k)}\right)^{T} W C_{y} W J\left(\theta^{(k)}\right)\left(J\left(\theta^{(k)}\right)^{T} W J\left(\theta^{(k)}\right)\right)^{-1}
$$

By choosing $W=C_{y}^{-1}$ the expression simplifies to:

$$
C_{W L S}=\left(J\left(\theta^{(k)}\right)^{T} C_{y}^{-1} J\left(\theta^{(k)}\right)\right)^{-1}
$$

It can be shown that, among all possible positive definite choices for $\mathrm{W}$, the best one is $W=C_{y}^{-1}$ since this minimizes the covariance matrix (Pintelon \& Schoukens, 2001). These expressions depend on the covariance matrix of the noise $C_{y}$. In practice this knowledge should be obtained from measurements. In this work a sample covariance matrix obtained 
from repeated measurements is used. It can be shown that in this case the covariance matrix of the estimates is given by (Schoukens et al., 1997):

$$
C_{W L S}=\frac{M-1}{M-5}\left(J\left(\theta^{(k)}\right)^{T} C_{y}^{-1} J\left(\theta^{(k)}\right)\right)^{-1}
$$

where $M$ is the number of polarization curves that are measured. At least 11 experiments have to be performed to use this expression (Schoukens et al., 1997).

After SVD of $C_{y}^{-1} J$ this equation becomes:

$$
C_{W L S}=V \Sigma^{-2} V^{T} \frac{M-1}{M-5}
$$

In this work the Gauss-Newton and Levenberg-Marquardt methods are implemented. The fitting starts with the Gauss-Newton method, but when the cost function is no longer decreasing after an iteration, it switches to Levenberg-Marquardt. The reason for starting with the Gauss-Newton method is that this method usually converges more rapidly than the Levenberg-Marquardt algorithm.

\subsection{A statistical evaluation}

After the determination of the best-fit parameter values, the validity of the selected model should be tested: does this model describe the available data properly or are there still indications that a part of the data is not explained by the model, indicating remaining model errors? We need the means to assess whether or not the model is appropriate, that is, we need to test the goodness-of-fit against some useful statistical standard.

With the best-fit-parameters, the modeled data is calculated and compared with the experimental data. Also, the difference between the experimental and calculated data is plotted. If the model describes the experiment appropriately, this difference should lie in the confidence band. This interval is defined by \pm two times the experimental standard deviation of the current, calculated from a series of repeated experiments. When performing multiple experiments, $95 \%$ of the experiments are expected to fall in this interval.

If the statistical evaluation does not show a good agreement between model and experiments, the model cannot be accepted indicating that any of the modeling steps is not well designed. In that case, we have to go back to the previous modeling steps: the experimental study, the proposed model (reaction or transport model) or/and the fitting procedure, and check their performance and the assumptions and simplifications that have been made to develop every step of the modeling methodology. Adjustments or possible alternatives must then be proposed.

An important message is that the fitting of parameters is not the end-all of parameter estimation. To be genuinely useful, a fitting procedure should provide (i) parameters, (ii) error estimates on the parameters, and (iii) a statistical measure of goodness-of-fit. When the third item suggests that the model is an unlikely match to the data, then items (i) and (ii) are probably worthless. Unfortunately, many practitioners of parameter estimation never proceed beyond item (i). They deem a fit acceptable if a graph of data and model "look good". This approach is known as chi-by-eye and should definitively be avoided. 


\section{Analytical modeling of LSV/RDE experiments}

\subsection{The experimental procedure}

\subsubsection{Composition of the electrolytes}

The chemicals used for the study of the hexaammineruthenium (III)/(II) redox reaction are: $\left[\mathrm{Ru}\left(\mathrm{NH}_{3}\right)_{6}\right] \mathrm{Cl}_{3}$ (Sigma-Aldrich, 98\%), $\mathrm{Na}_{2} \mathrm{HPO}_{4} \cdot 2 \mathrm{H}_{2} \mathrm{O}$ and $\mathrm{NaH}_{2} \mathrm{PO}_{4} \cdot 12 \mathrm{H}_{2} \mathrm{O}$ (both ProLabo A.R.). Solutions are made with once-distilled and deionized water. A $0.1 \mathrm{M}$ phosphate buffer $\mathrm{pH} 7$ solution is used as the supporting electrolyte and the concentration of the electroactive species hexaammineruthenium (III) is $0.001 \mathrm{M}$. The following chemicals are used for the ferri/ferrocyanide reaction: $\mathrm{K}_{4}\left[\mathrm{Fe}(\mathrm{CN})_{6}\right] \cdot 3 \mathrm{H}_{2} \mathrm{O}, \mathrm{K}_{3}\left[\mathrm{Fe}(\mathrm{CN})_{6}\right]$ and $\mathrm{KCl}$ (all Merck P.A.). The supporting electrolyte is a $1 \mathrm{M} \mathrm{KCl}$ solution and the concentrations of the electroactive components ferri/ferrocyanide are $0.005 \mathrm{M}$. In that way, a negligible migration flux, constant activity and diffusion coefficients of the electroactive species, a low electrolyte resistance and a uniform current distribution are assumed for both electrochemical systems.

\subsubsection{Experimental set-up}

A typical three electrode set-up is used for the LSV/RDE experiments (Bamford \& Compton, 1986; Diard et al., 1996). The electrochemical cell contains a $\mathrm{Ag} / \mathrm{AgCl}$ reference electrode (Schott-Geräte), a rotating disk working electrode and a platinum grid as counter electrode. A platinum RDE is used for the ferri/ferrocyanide system and a gold RDE for the hexaamineruthenium (III)/(II) system. The RDE electrodes are fabricated by embedding a $4 \mathrm{~mm}$ diameter polycrystalline platinum or gold rod in an insulating mantle of polyvinylidenefluoride.

The electrode is rotated by an RDE control system of Autolab. For the hexaammineruthenium (III)/(II) reaction, the rotation speed is set to 500 and $1000 \mathrm{rpm}$. In the experiments with the ferri/ferrocyanide system, the rotation speeds are 300, 1000, 1500 and $2000 \mathrm{rpm}$. The voltammograms are measured using a high resolution galvanostat/potentiostat PGSTAT100 (Autolab Instruments) of Ecochemie, controlled by the GPES 4.8 or the Nova 1.5 softwares. In the LSV experiments, the potential is swept from 0.3 to $-0.4 \mathrm{~V}$ vs NHE for the $\mathrm{Ru}\left(\mathrm{NH}_{3}\right)_{6}^{+3}$ / $\mathrm{Ru}\left(\mathrm{NH}_{3}\right)_{6}^{+2}$ reaction and from 0.8 to $0.2 \mathrm{~V}$ vs NHE for the $\mathrm{Fe}(\mathrm{CN})_{6}^{-3} / \mathrm{Fe}(\mathrm{CN})_{6}^{-4}$ reaction. The scan rate is taken constant at $1 \mathrm{mV} / \mathrm{s}$. The step potential is set to $0.00015 \mathrm{~V}$, in this way a maximum number of data points is measured.

All measurements are performed in a $200 \mathrm{ml}$ glass electrolytic cell, thermostatted at $25 \pm$ $0.5^{\circ} \mathrm{C}$ using a water jacket connected to a thermostat bath (Lauda RE304). Prior to the measurements, the electrolyte is deoxygenated by bubbling with nitrogen gas (Air Liquide) for $10 \mathrm{~min}$, while during the experiment a nitrogen blanket is maintained over the cell. This results in a substantial flattening of the reduction plateau of both the ferricyanide and the hexaamineruthenium (III).

\subsubsection{Electrode pretreatment}

The reproducibility of the measurements is strongly increased by means of applying the following pretreatment of the working electrode surface: 1) mechanical polishing of the electrode on a rotating disk (Struers DP10, on cloth), successively using diamond paste of $7 \mu \mathrm{m}$ and $1 \mu \mathrm{m}$ grain size (Struers) for the Pt electrode, and $9 \mu \mathrm{m}, 3 \mu \mathrm{m}$ diamond paste and 
$0.04 \mu \mathrm{m} \mathrm{Al}_{2} \mathrm{O}_{3}$ paste (Struers) for the Au electrode; 2) ultrasonic rinsing with deionized water followed by degreasing with chloroform, also in an ultrasonic bath (Elma model T470/H); 3) four cyclic voltammograms are performed before each experiment in order to remove oxide and trace contaminants from the metallic surface (Robertson et al., 1988). During the cyclic voltammetry measurements, the potential is swept between $-0.35 \mathrm{~V}$ to $1.45 \mathrm{~V}$ vs $\mathrm{Ag} / \mathrm{AgCl}$ at a scan velocity of $50 \mathrm{mV} / \mathrm{s}$ on the Au electrode (hexaammineruthenium (III)/(II) system) and between +0.55 and $-0.45 \mathrm{~V}$ vs $\mathrm{Ag} / \mathrm{AgCl}$ at a scan velocity of $10 \mathrm{mV} / \mathrm{s}$, on the Pt electrode (ferri/ferrocyanide system).

\subsection{Modeling the ferri/ferrocyanide redox reaction}

The simplest electrochemical reactions, which can be found among the different kinds of electrode processes, are those where electrons are exchanged across the interface by flipping oxidation states of transition metal ions in the electrolyte adjacent to the electrode surface (Bamford \& Compton, 1986), i.e. an ET (electron transfer) mechanism. The electrode acts as the source or sink of electrons for the redox reaction and is supposed to be inert. The reduction of ferricyanide to ferrocyanide (Angell \& Dickinson, 1972; Bamford \& Compton, 1986; Bruce et al., 1994; Iwasita et al., 1983) is an example of such a mechanism:

$$
\mathrm{Fe}(\mathrm{CN})_{6}^{-3}+e^{-} \rightleftharpoons \mathrm{Fe}(\mathrm{CN})_{6}^{-4}
$$

\subsubsection{Results of the experimental study}

A set of equivalent polarization curves of the ferri/ferrocyanide reaction is used as the experimental data, which is the input to the model methodology. As advised in (Tourwé et al., 2006), 11 voltammograms are measured under identical conditions for every rotation speed. The resulting voltammograms for the $\mathrm{Fe}(\mathrm{CN})_{6}^{-3} / \mathrm{Fe}(\mathrm{CN})_{6}^{-3}$ system are shown in Figure 3 .

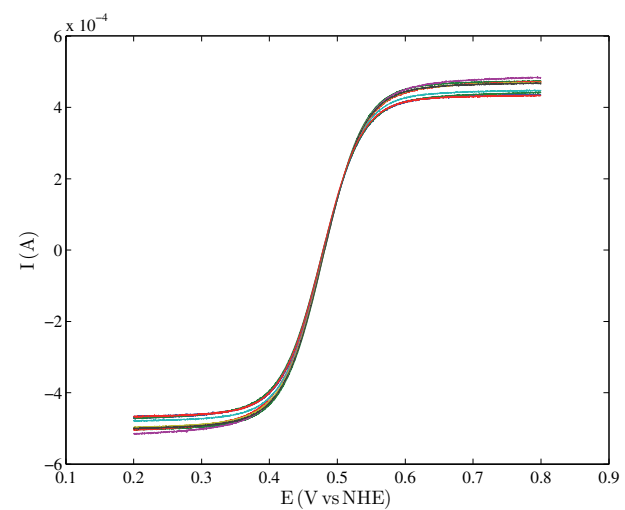

Fig. 3. Voltammograms of the reduction/oxidation of $0.005 \mathrm{M}$ ferri/ferrocyanide in $1 \mathrm{M} \mathrm{KCl}$, at $2000 \mathrm{rpm}$.

\subsubsection{The analytical expression for the current as a function of the potential}

The analytical expression is derived for the reduction/oxidation of ferri/ferrocyanide (reaction (23)). The basic equations that describe this mechanism, when studied under 
steady-state conditions on a rotating disk electrode, are:

$$
\begin{gathered}
v=K_{o x} C_{r e d}(0)-K_{r e d} C_{o x}(0) \\
I=i S=i_{f} S=F S v \\
v=v_{o x / r e d}=-m_{o x}\left[C_{o x}^{*}-C_{o x}(0)\right]=m_{\text {red }}\left[C_{r e d}^{*}-C_{r e d}(0)\right] \\
i_{\text {lim }, o x / \text { red }}= \pm F m_{r e d / o x} C_{r e d / o x}^{*}= \pm 0.620 F D_{\text {red } / o x}^{2 / 3} v^{-1 / 6} \omega^{1 / 2} C_{r e d / o x}^{*} \\
K_{o x / r e d}=k_{o x / r e d} \exp \frac{ \pm \alpha_{o x / r e d} n F E}{R T}
\end{gathered}
$$

From this set of equations an expression for the current as a function of the potential, the experimental parameters and the model parameters can be derived. Considering $\alpha_{o x}+\alpha_{r e d}=$ 1 , the model parameters are $D_{r e d}, D_{o x}, k_{o x}, k_{r e d}$ and $\alpha_{o x}$. The experimental parameters are $C_{r e d}^{*}, C_{o x}^{*}, S, n$ and $T$, which are all known. $v$ and $\omega$ are eliminated in the expression for the current, by using the limiting current density as a model parameter, rather than the diffusion coefficient. Solving this set of equations leads to the following current-potential relation (Tourwé et al., 2007):

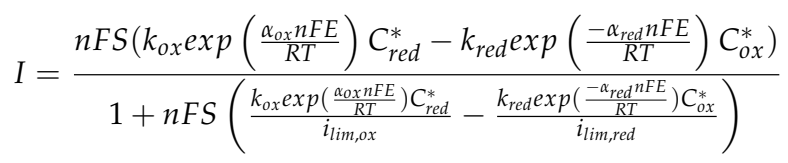

\subsubsection{The fitting results and their statistical evaluation}

For the ferri/ferrocyanide system, the analytical model is based on 5 parameters: the rate constant of the reduction reaction, $k_{r e d}$, the rate constant of the oxidation reaction, $k_{o x}$, the charge transfer coefficient, $\alpha_{o x}$, the reduction limiting current density, $i_{l i m, \text { red }}$ and the oxidation limiting current density, $i_{l i m, o x}$. The mathematical expression for the current as a function of the potential (Eq. (29)) is fitted to the mean of the equivalent experimental voltammograms, using the method described previously.

Four rotation speeds are considered for the study of the ferri/ferrocyanide redox reaction: 300, 1000, 1500 and $2000 \mathrm{rpm}$. Figure 4.a presents a comparison between the mean experimental voltammogram at $2000 \mathrm{rpm}$ and the modeled voltammogram, calculated with the best-fit-parameters, and the $95 \%$ confidence interval $\pm 2 \sigma$, with $\sigma$ the standard deviation of the current in the set of experimental curves. The difference between the experimental and the modeled curve (Figure 4.b) lies in the 95\% confidence band. This means that the model is able to describe the experiments appropiately.

In order to check the validity of the analytical modeling to estimate the model parameters, an evaluation of the parameter values determined at different rotation speeds is carried out. In Figure 5 the $95 \%$ confidence intervals for the best fit-parameters at four rotation speeds are compared. It can be seen that for all parameters the $95 \%$ confidence intervals obtained at the different rotation speeds overlap with the interval for the lowest rotation speed (300 $\mathrm{rpm}$ ). The latter is, however, much larger than those obtained for the higher rotation speeds. This is due to the fact that at lower rotation speeds mass transfer rapidly becomes the rate determining step. The potential region where charge transfer influences the reaction rate is smaller than for the other rotation speeds and, as a consequence, the charge transfer 


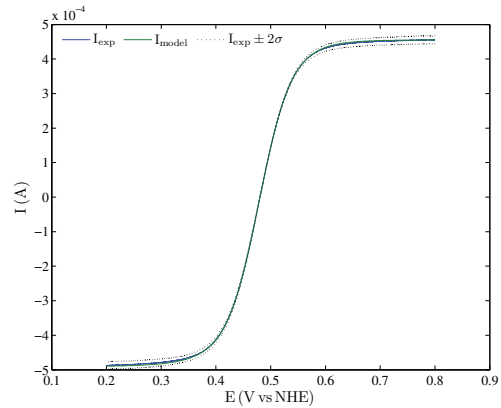

(a)

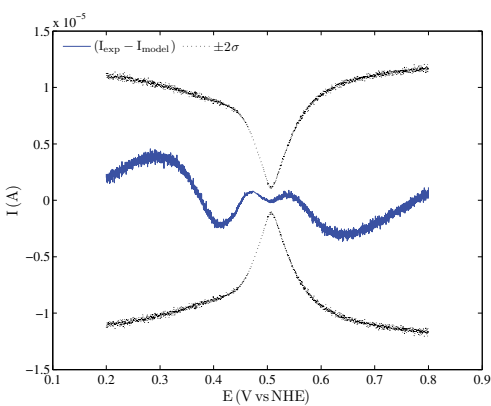

(b)

Fig. 4. Modeling the reduction/oxidation of ferri/ferrocyanide: (a) Comparison of the modeled curve and the mean experimental curve, at $2000 \mathrm{rpm}$. (b) Difference between the experiments and the model within the $95 \%$ confidence interval.

parameters are determined less accurately. This does not imply that the results at this lowest rotation speed have to be rejected. When calculating the best estimates of the parameters, the higher uncertainty is taken into account. The $95 \%$ confidence intervals for $k_{o x}, k_{r e d}$ and $\alpha_{o x}$ obtained for the intermediate rotation speeds (1000, 1500 and $2000 \mathrm{rpm}$ ) overlap, indicating that the results are independent of the rotation speed.

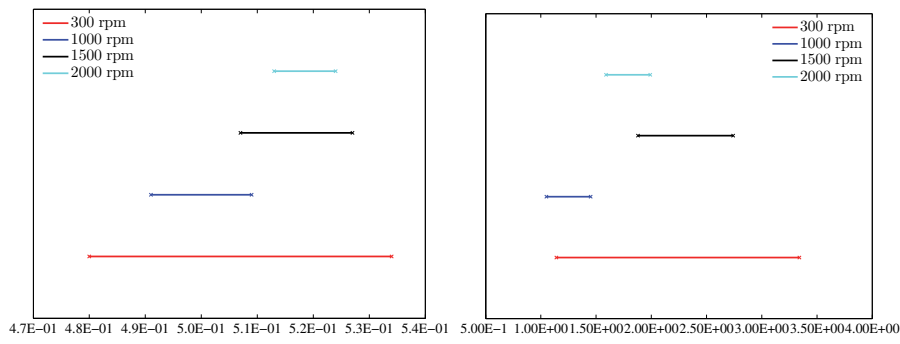

(a) $\alpha_{o x}$

(b) $k_{\text {red }}(\mathrm{m} / \mathrm{s})$

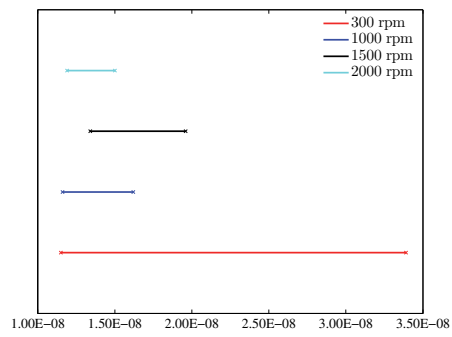

(c) $k_{o x}(\mathrm{~m} / \mathrm{s})$

Fig. 5. Comparison of the $95 \%$ confidence intervals of the charge transfer parameters obtained at $300 \mathrm{rpm}, 1000 \mathrm{rpm}, 1500 \mathrm{rpm}$ and $2000 \mathrm{rpm}$. 
The best-fit estimates of the charge transfer parameters are calculated as the weighted mean of the values obtained at the four rotation speeds taken into consideration. Introducing weighting factors helps to adjust the impact of one result on the mean with regard to their level of uncertainty. The maximum likelihood estimate of normally distributed variables $x_{i}$ with different variances $\sigma_{i}^{2}$ and the same mean value $\mu$ is given by (Sorenson, 1980):

$$
\mu=\frac{\sum_{i} \frac{x_{i}}{\sigma_{i}^{2}}}{\sum_{i} \frac{1}{\sigma_{i}^{2}}}
$$

This results in the following values for the charge transfer coefficient and the rate constants: $\alpha_{o x}=5.14 \mathrm{E}-01 \pm 1.28 \mathrm{E}-02, k_{o x}=1.41 \mathrm{E}-08 \mathrm{~m} / \mathrm{s} \pm 4.50 \mathrm{E}-09 \mathrm{~m} / \mathrm{s}$ and $k_{r e d}=1.61 \mathrm{E}+00 \mathrm{~m} / \mathrm{s} \pm$ $4.82 \mathrm{E}-01 \mathrm{~m} / \mathrm{s}$.

The diffusion coefficients can be calculated from the Levich equation (Eq. 8). Using the values of $i_{\text {lim,red }}$ and $i_{\text {lim,ox }}$, determined for 300, 1000, 1500 and $2000 \mathrm{rpm}$, this results in $D_{\text {red }}=$ $8.07 \mathrm{E}-10 \mathrm{~m}^{2} / \mathrm{s} \pm 2.23 \mathrm{E}-17 \mathrm{~m}^{2} / \mathrm{s}$ and $D_{o x}=8.31 \mathrm{E}-10 \mathrm{~m}^{2} / \mathrm{s} \pm 2.75 \mathrm{E}-17 \mathrm{~m}^{2} / \mathrm{s}$. The obtained parameter values compare well with the values presented in literature (Angell \& Dickinson, 1972; Beriet \& Pletcher, 1993; Bruce et al., 1994; Jahn \& Vielstich, 1962).

\subsection{The analytical modeling of the hexaammineruthenium (III)/(II) redox reaction}

The reduction of hexaammineruthenium (III) to hexaammineruthenium (II) is extensively described in literature (Beriet \& Pletcher, 1994; Deakin et al., 1985; Elson et al., 1975; Khoshtariya et al., 2003; Marken et al., 1995; Muzikar \& Fawcett, 2006) as a one electron transfer reaction, i.e.,

$$
\mathrm{Ru}\left(\mathrm{NH}_{3}\right)_{6}^{+3}+e^{-} \rightleftharpoons \mathrm{Ru}\left(\mathrm{NH}_{3}\right)_{6}^{+2}
$$

Due to the fact that the hexaamineruthenium (II) complex is not stable in solution, the electrochemical reaction (31) is studied here only in the direction of the reduction, with the hexaammineruthenium (III) being the only electroactive species initially present in the electrolyte.

For the reduction of $\mathrm{Ru}\left(\mathrm{NH}_{3}\right)_{6}^{+3}$ to $\mathrm{Ru}\left(\mathrm{NH}_{3}\right)_{6}^{+2}$, the mathematical expression describing the reaction mechanism is obtained, leaving out the oxidation parameters. The current-potential relation is formulated with the following expression:

$$
I=\frac{-n F S k_{r e d} \exp \left(\frac{-\alpha_{r e d} n F E}{R T}\right) C_{o x}^{*}}{i_{\text {lim,red }}-n F S k_{\text {red }} \exp \left(\frac{-\alpha_{r e d} n F E}{R T}\right) C_{o x}^{*}}
$$

For the analytical modeling of the reaction, also 11 identical experimental curves are performed, at 500 and $1000 \mathrm{rpm}$. In the reduction of $\mathrm{Ru}\left(\mathrm{NH}_{3}\right)_{6}^{+3}$, an unexpected variation of the current values in the region of the limiting current is observed. This behavior differs from the characteristic curve of an ET mechanism and might be due to additional reactions in the supporting electrolyte. This contribution of the supporting electrolyte is measured and subtracted from the experimental polarization curves.

In Figure 6 it can be seen that the difference between the experimental curve and the modeled curve, at $500 \mathrm{rpm}$, lies in the $95 \%$ confidence band. At this point we could accept that the model is appropiate to describe the experiments, but an evaluation of the estimated values 


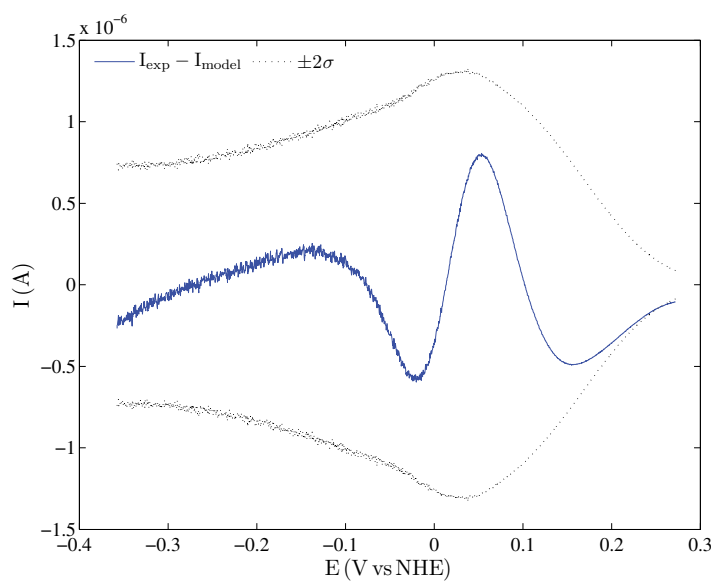

Fig. 6. Difference between the mean experimental and the modeled curves, at $500 \mathrm{rpm}$, within the $95 \%$ confidence band.

of the model parameters must be done. The model parameters are fitted for the experimental data obtained at two different rotation speeds. The best-fit values and their standard deviation obtained for $500 \mathrm{rpm}$ and $1000 \mathrm{rpm}$ are shown in Table 1. The values of the charge transfer coefficient are slightly different. In the case of the rate constant, the best-fit values at different rotation speeds present significant differences. Thus, the estimated values are not consistent with the results expected for the proposed model: while the limiting current density is proportional to the square root of $\omega$, the values of the charge transfer parameters must be independent of the rotation speed.

The different results of the estimated parameters at the two rotation speeds could be due to interfering reactions in the supporting electrolyte or, more likely, to the oxidation of hexaamineruthenium (II) to hexaamineruthenium (III), occurring at the initial potentials of the polarization curve. The oxidation half-reaction is not taken in consideration in the formulation of the analytical current-potential expression; this could have an influence on the parameter estimation. According to our modeling strategy, a better estimation of the model parameters needs further investigation on the treatment of the experimental data and/or the formulation of the reaction mechanism. Present research is devoted to this topic.

\begin{tabular}{|l|c|c|c|c|}
\hline \multirow{2}{*}{ Model parameters } & \multicolumn{2}{|c|}{$500 \mathrm{rpm}$} & \multicolumn{2}{c|}{$1000 \mathrm{rpm}$} \\
\cline { 2 - 5 } & Estimate & Std & Estimate & Std \\
\hline$\alpha_{\text {red }}$ & $7.67 \mathrm{E}-01$ & $2.95 \mathrm{E}-03$ & $7.49 \mathrm{E}-01$ & $2.15 \mathrm{E}-03$ \\
\hline$k_{\text {red }}(\mathrm{m} / \mathrm{s})$ & $6.17 \mathrm{E}-05$ & $3.36 \mathrm{E}-07$ & $7.14 \mathrm{E}-05$ & $3.46 \mathrm{E}-07$ \\
\hline$i_{\text {lim,red }}\left(\mathrm{A} / \mathrm{m}^{2}\right)$ & $-3.29 \mathrm{E}+00$ & $1.51 \mathrm{E}-03$ & $-4.54 \mathrm{E}+00$ & $2.20 \mathrm{E}-03$ \\
\hline
\end{tabular}

Table 1. Best-fit parameters and their standard deviation for $500 \mathrm{rpm}$ and $1000 \mathrm{rpm}$.

\section{The inverted rotating disk electrode (IRDE) reactor}

The combination of linear sweep voltammetry with an RDE is a powerful tool to study electrochemical reactions. One limitation to the technique is the presence of gas in some electrochemical systems. The inverted rotating disk electrode (IRDE) is designed to tackle 
this problem. It allows an investigation of gas evolving reactions, preventing the presence of gas from being an obstacle to a quantitative modeling.

When the RDE set-up is used for the mechanistic study of gas evolution reactions, the formed gas bubbles tend to stick to the downward facing electrode surface and, hence, shield the active electrode surface. In contrast to the classical RDE configuration where the rotating disk is downward facing and positioned at the top of the electrochemical cell, in the IRDE configuration the rotating disk is now placed at the bottom of the cell, facing upwards. As a result, the generated bubbles rise freely and are no longer shielding the electrode surface. The bubbles are detached from the electrode by buoyancy or swept away by the rotational movement of the electrode.

Many industrial electrochemical systems deal with gas evolution, such as, for example, the water electrolysis, the chlorine and chlorate production or the side reactions during the electrowinning or electrodeposition of metals.

\subsection{Design and construction of the IRDE reactor}

The electrochemical cell consists of a cylindrical vessel of $74 \mathrm{~mm}$ diameter and $200 \mathrm{~mm}$ height, with a square water jacket around it. A schematic illustration of the IRDE design is presented in Figure 7 and a picture of the IRDE set-up is shown in Figure 8. The height of the working electrode protruding into the electrolyte is $30 \mathrm{~mm}$. The counter and the reference electrode are located in the upper part of the cell, far away from the rotating electrode in order not to disturb the flow field near the working electrode. Because of the positioning of the reference electrode vs the working electrode, the current-potential data needs to be corrected for the ohmic drop. After emptying the cell, the vessel itself can be removed permitting an easy exchange of the working electrode. The cell is entirely made of plexiglas (polymethylmethacrylate or PMMA) to ensure full optical access to the cell. In this way, the gas bubbles can be characterized in-situ (e.g. bubble size, rise velocity, etc.) by means of optical imaging techniques.

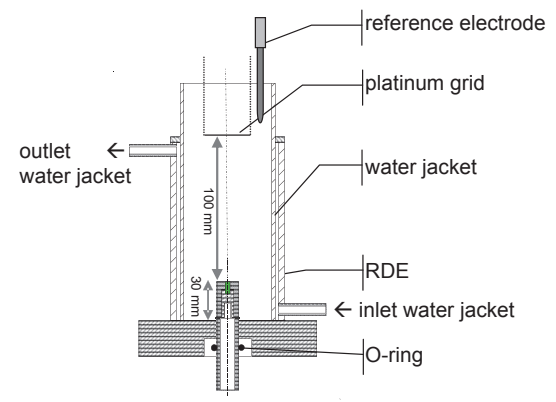

(a) Front view

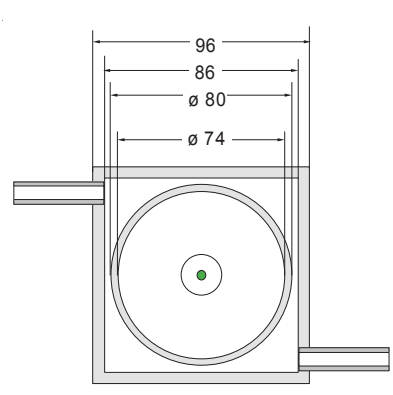

(b) Top view

Fig. 7. Schematic illustration of the IRDE cell. 


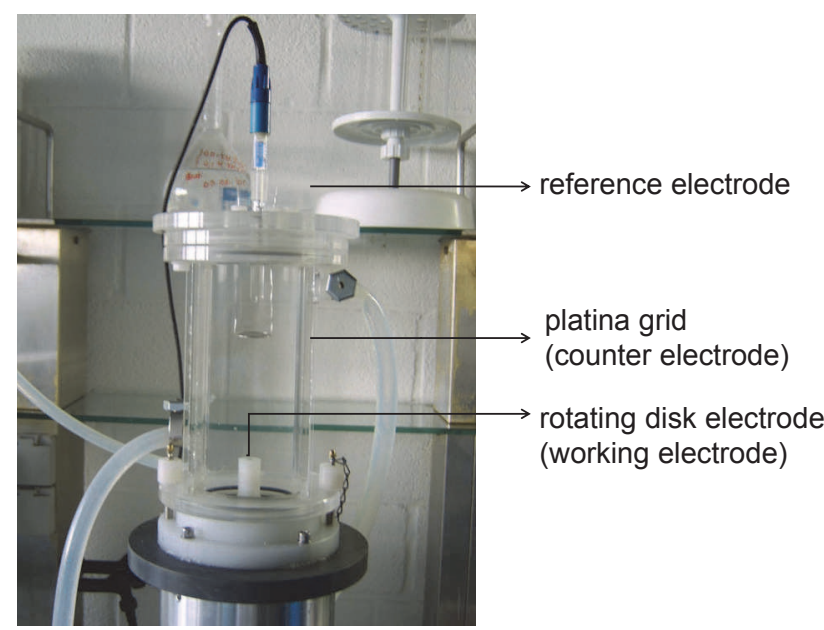

Fig. 8. Picture of the IRDE cell.

\subsection{Evaluation of the IRDE reactor for kinetic studies of electrochemical reactions}

Although the change in position of the working electrode from the top to the bottom of the cell seems to be a minor modification, it has to be examined whether the characteristics of mass transfer governing the RDE reactor are also valid for the IRDE reactor. To this purpose, the ferri/ferrocyanide redox system is used. Since theoretically charge transfer parameters are independent of the hydrodynamics, the same parameter values must be found in both the RDE and IRDE configuration. The equations of mass transfer, used for kinetic studies in an RDE configuration, are based on an analytical expression of the velocity field with the assumption of an infinite large electrode disk and bath dimensions. In practice, however, we deal with a finite sized rotating disk and a confined electrochemical cell. Although it is generally accepted that the analytical solution of the flow field derived by Levich (Newman, 1973) describes the velocity field near the electrode surface well, it is nevertheless not evident that the velocity field is described with the same accuracy for the IRDE configuration as for the RDE configuration.

The analytical modeling utilized for the RDE configuration is applied to the IRDE reactor. The experimental procedure described in section 4.1 is used for the LSV experiments of the ferri/ferrocyanide redox reaction on the IRDE. Eq. 29 is fitted to the mean polarization curves, obtained at 300, 1000 and $1500 \mathrm{rpm}$. The mean polarization curve is calculated from 11 independently recorded polarization curves. The $95 \%$ confidence intervals of $k_{o x}, k_{r e d}$ and $\alpha_{o x}$ obtained at 300, 1000 and $1500 \mathrm{rpm}$ perfectly overlap (not shown). This means that the values of the charge transfer parameters, $k_{o x}, k_{r e d}$ and $\alpha_{o x}$, are independent of the rotation speed, as expected theoretically. The calculated weighted mean values of the model parameters for charge transfer, $k_{o x}, k_{\text {red }}$ and $\alpha_{o x}$, are respectively $1.03 \mathrm{E}-08 \mathrm{~m} / \mathrm{s} \pm 1.31 \mathrm{E}-09 \mathrm{~m} / \mathrm{s}, 9.12 \mathrm{E}-01 \pm$ $1.25 \mathrm{E}-01 \mathrm{~m} / \mathrm{s}$ and $5.39 \mathrm{E}-01 \pm 7.06 \mathrm{E}-03$. 
In Figure 9, the weighted mean values of the charge transfer parameters and their $95 \%$ confidence interval, obtained in the IRDE and the RDE reactors, are compared. It can be seen that the confidence intervals of $k_{o x}$ perfectly overlap, while a small deviation between the confidence intervals is observed for $k_{\text {red }}$ and $\alpha_{o x}$. However, the deviation between the confidence interval is smaller than $1 \%$ for $\alpha_{0 x}$ and smaller than $5 \%$ for $k_{\text {red }}$. The deviation between the confidence intervals is determined by the difference between the maximum and the minimum values of the respective confidence intervals obtained in the RDE and the IRDE configurations, normalized to the mean value of the respective model parameter obtained in the RDE configuration. The values lie sufficiently close that it is reasonable to assume that the mass transfer characteristics of the RDE are also valid for the IRDE. The estimation of the charge transfer parameters does not depend on the hydrodynamics and mass transport within the electrochemical cell, which in its turn demonstrates that the IRDE is a suitable tool for the mechanistic study of the electrochemical reactions. Moreover, the differences between the values of the rate constants estimated for the RDE and the IRDE reactors narrow the window of values reported in literature (Angell \& Dickinson, 1972; Beriet \& Pletcher, 1993; Bruce et al., 1994; Jahn \& Vielstich, 1962).

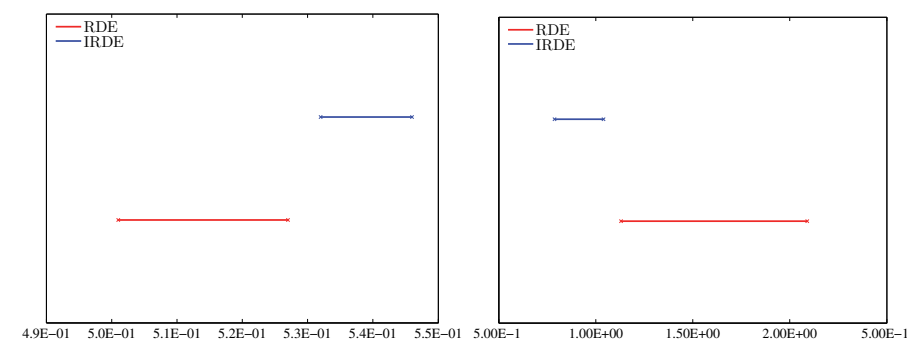

(a) $\alpha_{o x}$

(b) $k_{\text {red }}(\mathrm{m} / \mathrm{s})$

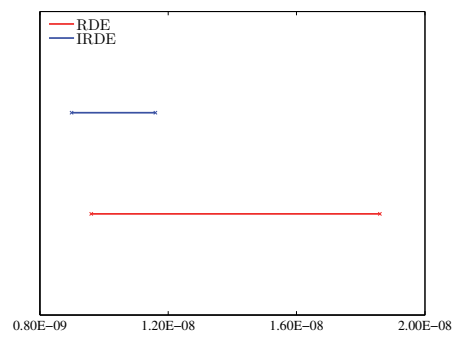

(c) $k_{o x}(\mathrm{~m} / \mathrm{s})$

Fig. 9. Comparison of the $95 \%$ confidence intervals of the weighted mean of the charge transfer parameters obtained in the IRDE and the RDE configurations.

\section{Towards the identification and quantification of characteristic parameters of complex electrochemical systems}

In general, an accurate and fully statistical founded solution is the aim of all kinetic and mechanistic studies of electrochemical systems. The proposed analytical fitting model is 
applied to one electron transfer reactions. Yet the fitting can be extended to other reaction steps, such as chemical or adsorption reactions, or to other measurement techniques, such as, for example, electrochemical impedance spectroscopy, provided that they can be analytically formulated.

However, in many cases the electrochemical systems present more complicated reaction mechanisms, including multiple electrochemical or chemical reactions. The reaction model is thus too complex to be translated into analytical equations. Besides, when gas bubbles are involved in the electrochemical process, a new transport model describing the two-phase mass transport is designed (Maciel et al., 2009; Nierhaus et al., 2009; Van Damme et al., 2010). Modeling these complex systems requires a numerical approach. In our group, focus is put on the development of numerical models for such complex systems. Nevertheless, in order to achieve a statistical and accurate parameter estimation, it is clear that also a numerical fitting procedure has to be introduced. This aspect is under development at present in our group.

\section{Conclusions}

The strength of the analytical fitting model to quantify the kinetic parameters of an electrochemical reaction is shown. The coupling of statistically founded parameter estimation techniques with LSV/RDE experiments is an important innovative point of the modeling strategy.

The fitting methodology requires the proposition of an appropriate mechanism for the studied reaction and its mathematical translation into an expression that analytically describes the voltammogram. This expression depends on the mass and charge transfer parameters of the reaction (rate constants, transfer coefficients and diffusion coefficients). Powerful parameter estimation algorithms are used in the data fitting tool to adjust the values of the model parameters in order to obtain a good agreement between experimental and modeled data. The values of the model parameters that give rise to the best match, characterize the system quantitatively. Moreover, this method provides error estimates of the obtained parameter values. However, it is only after a statistical evaluation of the obtained results, that it is decided whether the model is able to describe the experiments.

The application of the analytical modeling for the study of the ferri/ferrocyanide reaction with LSV/RDE experiments demonstrate that the modeling methodology is valid to extract the quantitative mechanism of an electrochemical reaction. In the case of the hexaammineruthenium (III)/(II) reaction, however, the results of the analytical modeling point out the importance of a correct formulation of the reaction mechanism.

The IRDE reactor is built to facilitate the study of electrochemical gas evolution reactions. It offers the advantages of the classical RDE set-up, such as well-defined hydrodynamics and mass transport over a wide range of rotation speeds, while the gas bubbles can rise freely and do not shield the electrode surface. It is demonstrated that the IRDE configuration is valid for kinetic and mechanistic investigations of electrochemical reactions.

It has to be emphasized that for the existing fitting procedure the proposed reaction model describing the reactions taking place must be translated into an analytical equation. It is clear that in the presence of, for example, chemical reactions or gas bubbles, an analytical solution does not exist anymore. Therefore, for further modeling studies a fitting tool that makes use of numerical calculation procedures needs to be developed. 


\section{Acknowledgments}

The authors thank the Flemish Institute for support of Scientific-Technological Research in Industry (IWT) and Vrije Universiteit Brussel for the financial support.

\section{References}

Aerts, T., Tourwe, E., Pintelon, R., De Graeve, I. \& Terryn, H. (2011). Modelling of the porous anodizing of aluminium: Generation of experimental input data and optimization of the considered model, Surface \& Coatings Technology 205(19): 4388-4396.

Albery, W. \& Hitchman, M. (1971). Ring-Disc Electrodes, Clarendon Press.

Angell, D. \& Dickinson, T. (1972). Kinetics of ferrous/ferric and ferro/ferricyanide reactions at platinum and gold electrodes. 1. Kinetics at bare-metal surfaces, Journal of Electroanalytical Chemistry 35: 55-\&.

Aslam, M., Harrison, J. \& Small, C. (1980). The automation of electrode kinetics. 2: The application to metal-deposition, Electrochimica Acta 25(5): 657-668.

Bamford, C. \& Compton, R. (1986). Electrode Kinetics: Principles and Methodology. Comprehensive Chemical Kinetics, Vol. 26, Elsevier Science Publishers.

Beriet, C. \& Pletcher, D. (1993). A microelectrode study of the mechanism and kinetics of the ferro ferricyanide couple in aqueous media - the influence of the electrolyte and its concentration, Journal of Electroanalytical Chemistry 361: 93-101.

Beriet, C. \& Pletcher, D. (1994). A further microelectrode study of the influence of electrolyte concentration on the kinetics of redox couples, Journal of Electroanalytical Chemistry 375(1-2): 213-218.

Bortels, L., Van den Bossche, B. \& Deconinck, J. (1997). Analytical solution for the steady-state diffusion and migration. Application to the identification of Butler-Volmer electrode reaction parameters, Journal of Electroanalytical Chemistry 422(1-2): 161-167.

Bruce, P., Lisowskaoleksiak, A., Los, P. \& Vincent, C. (1994). Electrochemical impedance spectroscopy at an ultramicroelectrode, Journal of Electroanalytical Chemistry 367(1-2): 279-283.

Caster, D., Toman, J. \& Brown, S. (1983). Curve fitting of semiderivative linear scan voltammetric responses - Effect of reaction reversibility, Analytical Chemistry 55(13): 2143-2147.

Cowan, K. \& Harrison, J. (1980a). The automation of electrode kinetics. 3: The dissolution of $\mathrm{Mg}$ in $\mathrm{Cl}^{-}, \mathrm{F}^{-}$and $\mathrm{OH}^{-}$containing aqueous solutions, Electrochimica Acta 25(7): 899-912.

Cowan, K. \& Harrison, J. (1980b). Automation of electrode kinetics. 5: The dissolution of Al in $\mathrm{Cl}^{-}$and $\mathrm{F}^{-}$containing aqueous solutions, Electrochimica Acta 25(9): 1153-1163.

Deakin, M., Stutts, K. \& Wightman, R. (1985). The effect of pH on some outer-sphere electrode-reactions at carbon electrodes, Journal of Electroanalytical Chemistry 182(1): 113-122.

Denton, D., Harrison, J. \& Knowles, R. (1980). Automation of electrode kinetics. 4: The chlorine evolution reaction on a $\mathrm{RuO}_{2}-\mathrm{TiO}_{2}$ plate electrode, Electrochimica Acta 25(9): 1147-1152.

Diard, J.-P., Le Gorrec, B. \& Montella, C. (1996). Cinétique électrochimique, Hermann.

Elson, C., Itzkovitch, I., McKenney, J. \& Page, J. (1975). Electrochemistry of ruthenium ammine complexes, Canadian Journal of Chemistry-revue Canadienne De Chimie 53(19): 2922-2929. 
Fletcher, R. (1980). Practical Methods of Optimization, Vol. 1, John Wiley and Sons.

Gattrell, M., Park, J., MacDougall, B., Apte, J., McCarthy, S. \& Wu, C. (2004). Study of the mechanism of the vanadium $4+/ 5+$ redox reaction in acidic solutions, Journal of the Electrochemical Society 151(1): A123-A130.

Harrison, J. (1982a). Automation of electrode kinetic measurements. 7, Electrochimica Acta 27(8): 1113-1122.

Harrison, J. (1982b). Automation of electrode kinetic measurements. 8, Electrochimica Acta 27(8): 1123-1128.

Harrison, J. \& Small, C. (1980a). The automation of electrode kinetic measurements. 1: The instrumentation and the fitting of the data using a library of reaction schemes, Electrochimica Acta 25(4): 447-452.

Harrison, J. \& Small, C. (1980b). Automation of electrode kinetics. 6: Dissolution of $\mathrm{Pb}$ in $\mathrm{H}_{2} \mathrm{SO}_{4}$, Electrochimica Acta 25(9): 1165-1172.

Iwasita, T., Schmickler, W., Herrmann, J. \& Vogel, U. (1983). The kinetic-parameters of the $\mathrm{Fe}(\mathrm{CN})_{6}^{3-/ 4-}$ redox system - New results with the ring electrode in turbulent pipe-flow, Journal of the Electrochemical Society 130(10): 2026-2032.

Jahn, D. \& Vielstich, W. (1962). Rates of electrode processes by the rotating disk method, Journal of Electrochemical Society 109: 849-852.

Kelley, C. (1999). Iterative Methods for Optimization, SIAM.

Khoshtariya, D., Dolidze, T., Vertova, A., Longhi, M. \& Rondinini, S. (2003). The solvent friction mechanism for outer-sphere electron exchange at bare metal electrodes. the case of $\mathrm{au} / \mathrm{Ru}\left(\mathrm{NH}_{3}\right)_{6}^{3+/ 2+}$ redox system, Electrochemistry Communications 5(3): 241-245.

Levich, V. (1962). Physicochemical Hydrodynamics, Prentice-Hall.

Maciel, P., Nierhaus, T., Van Damme, S., Van Parys, H., Deconinck, J. \& Hubin, A. (2009). New model for gas evolving electrodes based on supersaturation, Electrochemistry Communications 11(4): 875-877.

Marken, F., Eklund, J. \& Compton, R. G. (1995). Voltammetry in the presence of ultrasound - can ultrasound modify heterogeneous electron-transfer kinetics?, Journal of Electroanalytical Chemistry 395(1-2): 335-339.

Muzikar, M. \& Fawcett, W. (2006). Medium effects for very fast electron transfer reactions at electrodes: the $\left[\mathrm{Ru}\left(\mathrm{NH}_{3}\right)_{6}\right]^{3+/ 2+}$ system in water, Journal of Physical Chemistry $B$ 110(6): 2710-2714.

Newman, J. (1973). Electrochemical Systems, Englewood Cliffs, Prentice-Hall.

Nierhaus, T., Van Parys, H., Dehaeck, S., van Beeck, J., Deconinck, H., Deconinck, J. \& Hubin, A. (2009). Simulation of the two-phase flow hydrodynamics in an IRDE reactor, Journal of the Electrochemical Society 156(9): P139-P148.

Norton, J. (1986). An Introduction to Identification, Academic Press.

Pintelon, R. \& Schoukens, J. (2001). System Identification - A Frequency Domain Approach, IEEE Press.

Pletcher, D. (1991). A First Course in Electrode Processes, Electrochemical Consultancy.

Press, H., Teukolsky, S., Vetterling, W. \& Flannery, B. (1988). Numerical Recipes in C - The Art of Scientific Computing, Cambridge University Press.

Robertson, B., Tribollet, B. \& Deslouis, C. (1988). Measurement of diffusion-coefficients by DC and EHD electrochemical methods, Journal of the Electrochemical Society 135(9): 2279-2284. 
Rocchini, G. (1992). The determination of the electrochemical parameters by the best-fitting with exponential polynomials, Corrosion Science 33(11): 1773-1788.

Rusling, J. (1984). Fitting tabulated current functions to linear-sweep voltammograms, Analytica Chimica Acta 162: 393-398.

Schoukens, J., Pintelon, R. \& Rolain, Y. (1997). Recent Advances in Total Least Squares Techniques and Errors-In-Variables Modeling, SIAM, chapter Maximum Likelihood Estimation of Errors-In-Variables Models Using a Sample Covariance Matrix Obtained from Small Data Sets, pp. 59-68.

Slichting, H. (1979). Boundary Layer Theory, McGraw-Hill.

Sorenson, H. (1980). Parameter Estimation - Principles and Problems, Marcel Dekker.

Thirsk, H. \& Harrison, J. (1972). A Guide to the Study of Electrode Kinetics, Academic Press.

Tourwé, E., Breugelmans, T., Pintelon, R. \& Hubin, A. (2007). Extraction of a quantitative reaction mechanism from linear sweep voltammograms obtained on a rotating disk electrode. Part II: Application to the redoxcouple $\mathrm{Fe}(\mathrm{CN})_{6}^{-3} / \mathrm{Fe}(\mathrm{CN})_{6}^{-4}$, Journal of Electroanalytical Chemistry 609(1): 1-7.

Tourwé, E., Pintelon, R. \& Hubin, A. (2006). Extraction of a quantitative reaction mechanism from linear sweep voltammograms obtained on a rotating disk electrode. Part I: Theory and validation, Journal of Electroanalytical Chemistry 594(1): 50-58.

Van Damme, S., Maciel, P., Van Parys, H., Deconinck, J., Hubin, A. \& Deconinck, H. (2010). Bubble nucleation algorithm for the simulation of gas evolving electrodes, Electrochemistry Communications 12(5): 664-667.

Van den Bossche, B., Bortels, L., Deconinck, J., Vandeputte, S. \& Hubin, A. (1995). Quasi-one-dimensional steady-state analysis of multi-ion electrochemical systems at a rotating disc electrode controlled by diffusion, migration, convection and homogeneous reactions, Journal of Electroanalytical Chemistry 397(1-2): 35-44.

Van den Bossche, B., Floridor, G., Deconinck, J., Van Den Winkel, P. \& Hubin, A. (2002). Steady-state and pulsed current multi-ion simulations for a thallium electrodeposition process, Journal of Electroanalytical Chemistry 531(1): 61-70.

Van Parys, H., Telias, G., Nedashkivskyi, V., Mollay, B., Vandendael, I., Van Damme, S., Deconinck, J. \& Hubin, A. (2010). On the modeling of electrochemical systems with simultaneous gas evolution. Case study: The zinc deposition mechanism, Electrochimica Acta 55(20): 5709-5718.

Van Parys, H., Tourwé, E., Breugelmans, T., Depauw, M., Deconinck, J. \& Hubin, A. (2008). Modeling of mass and charge transfer in an inverted rotating disk electrode (IRDE) reactor, Journal of Electroanalytical Chemistry 622(1): 44-50.

Vetter, K. (1967). Electrochemical Kinetics: Theoretical and Experimental Aspects, Academic Press.

Wang, J., Markovic, N. \& Adzic, R. (2004). Kinetic analysis of oxygen reduction on Pt(111) in acid solutions: Intrinsic kinetic parameters and anion adsorption effects, Journal of Physical Chemistry B 108(13): 4127-4133.

Yeum, K. \& Devereux, O. (1989). An iterative method for fitting complex electrode polarization curves, Corrosion 45(6): 478-487. 


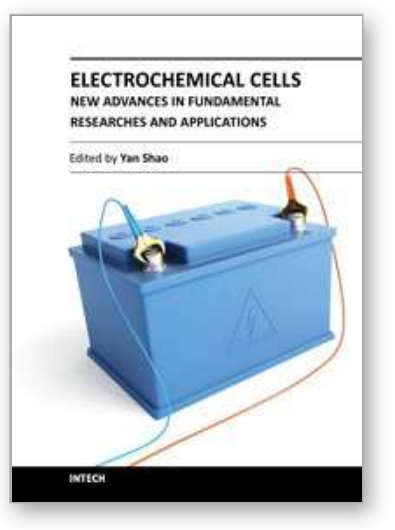

\section{Electrochemical Cells - New Advances in Fundamental Researches and Applications}

Edited by Dr. Yan Shao

ISBN 978-953-51-0032-4

Hard cover, 240 pages

Publisher InTech

Published online 07, March, 2012

Published in print edition March, 2012

Currently the research field of electrochemical cells is a hotspot for scientists and engineers working in advanced frontlines of micro-, nano- and bio-technologies, especially for improving our systems of energy generation and conversation, health care, and environmental protection. With the efforts from the authors and readers, the theoretical and practical development will continue to be advanced and expanded.

\section{How to reference}

In order to correctly reference this scholarly work, feel free to copy and paste the following:

Lucía Fernández Macía, Heidi Van Parys, Tom Breugelmans, Els Tourwé and Annick Hubin (2012). Modeling and Quantification of Electrochemical Reactions in RDE (Rotating Disk Electrode) and IRDE (Inverted Rotating Disk Electrode) Based Reactors, Electrochemical Cells - New Advances in Fundamental Researches and Applications, Dr. Yan Shao (Ed.), ISBN: 978-953-51-0032-4, InTech, Available from:

http://www.intechopen.com/books/electrochemical-cells-new-advances-in-fundamental-researches-andapplications/a-quantitative-investigation-of-electrochemical-reaction-mechanisms-in-the-rotating-diskelectrode-r

\section{INTECH}

open science | open minds

\section{InTech Europe}

University Campus STeP Ri

Slavka Krautzeka 83/A

51000 Rijeka, Croatia

Phone: +385 (51) 770447

Fax: +385 (51) 686166

www.intechopen.com

\section{InTech China}

Unit 405, Office Block, Hotel Equatorial Shanghai

No.65, Yan An Road (West), Shanghai, 200040, China

中国上海市延安西路65号上海国际贵都大饭店办公楼405单元

Phone: +86-21-62489820

Fax: +86-21-62489821 
(C) 2012 The Author(s). Licensee IntechOpen. This is an open access article distributed under the terms of the Creative Commons Attribution 3.0 License, which permits unrestricted use, distribution, and reproduction in any medium, provided the original work is properly cited. 